\title{
An Efficient Multi-resolution Spectrum Sensing Method for Cognitive Radio
}

\author{
Qiwei Zhang, Andre B.J. Kokkeler and Gerard J.M. Smit \\ Department of Electrical Engineering, Mathematics and Computer Science \\ University of Twente, Enschede, The Netherlands \\ Email: q.zhang@utwente.nl
}

\begin{abstract}
This paper presents a novel energy based multiresolution spectrum sensing technique. By applying an efficient flexible FFT, the proposed method can focus on a small part of the interested bands with finer resolutions at low computational cost. The hardware implementation of the algorithm has been considered. An experiment on a reconfigurable platform shows that the algorithm is not only computationally efficient but can also easy to be reconfigured easily and fast.
\end{abstract}

\section{INTRODUCTION}

The large demand for radio spectrum leaves little room to accommodate new wireless applications. However, recent studies have shown that most of the assigned radio spectrum is under-utilized. Cognitive Radio [1] is considered as a promising technology to address the paradox of spectrum scarcity and spectrum under-utilization. In Cognitive Radio, a spectrum sensing process locates the unused spectrum segments in a targeted spectrum pool. These segments will be used optimally without harmful interference to licensed users (users who have the legal license for the spectrum). This technology is called spectrum pooling [2]. Orthogonal frequency division multiplexing (OFDM) is proposed as the baseband transmission scheme due to its flexibility in the frequency domain. The additional benefit of OFDM is the reuse of the FFT module for energy based spectrum sensing.

Since spectrum sensing plays a vital role in Cognitive Radio to establish communications on unused spectrum, it has been an active research area in Cognitive Radio. On the signal processing level, two methods are often mentioned in literature: energy detection [3] and feature detection [4], [5]. Energy detection measures the signal power at a certain time interval and for a certain frequency band. The detection decision is based on a noise threshold. Energy detection can be implemented by averaging frequency bins of an FFT. Feature detection detects modulated signals by exploiting their hidden periodicity. Feature detection is more robust than energy detection in bad SNR scenarios, but it requires more computations [5]. Rather than solely relying on the sensing result of a single node, on the network level a collaborative sensing technique [6] can optimally combine the measurements from different nodes to produce a more robust result. In this paper, we focus on energy based spectrum sensing for the following reasons: 1) the computational complexity of feature detection makes it less favorable for real-time processing; 2) energy detection can provide the basis for a

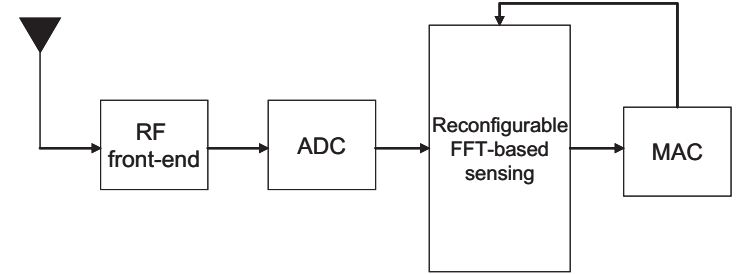

Fig. 1. Block diagram of reconfigurable FFT based multi-resolution sensing

network level collaborative sensing. The paper is organized as follows: Section 2 introduces the idea of multi-resolution spectrum sensing. We propose a multi-resolution spectrum sensing based on an efficient dynamically reconfigurable FFT in section 3. Section 4 discusses the implementation issues of the proposed algorithm and presents an experiment of the proposed algorithm on a reconfigurable platform. Section 5 concludes the paper.

\section{Multi-Resolution SPECTRUM SENSING}

The topic of multi-resolution for Cognitive Radio has been treated in the recent literature [7] and [8]. Although different methods have been applied in their papers, the basic idea is the same. The total bandwidth is first sensed using a coarse resolution. Fine resolution sensing is performed on a portion of the interested bands for Cognitive Radio. In such a way, Cognitive Radio avoids sensing the whole band at the maximum frequency resolution. Therefore, the sensing time is reduced and the power has been saved from unnecessary computations. Hur et al. [7] proposed a wavelet based multiresolution sensing technique in the analog domain. Neihart et al. [8] discuss an FFT based multi-resolution spectrum sensing for multiple antenna Cognitive Radio. In [8], the multiple antenna architecture enables parallel processing and thus reduces the sensing time. However, it increases the chip area and power consumption. Moreover, the mixer has to generate multiple frequencies for coarse resolution sensing and has to switch to a single frequency while starting fine resolution sensing.

In this paper, we assume a single antenna Cognitive Radio receiver (see figure 1) which can digitize the total targeted bandwidth of $B_{t o t}$ which is unlike the assumption in [8] where the total bandwidth has to be digitized multiple times into smaller blocks. The frequency resolution $f_{r}=\frac{B_{t o t}}{K}$, 


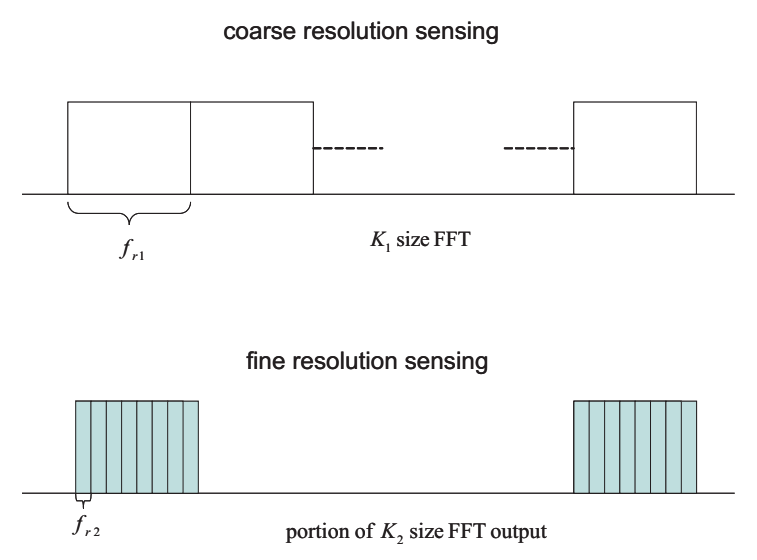

Fig. 2. An multi-resolution sensing example

where $K$ is the size of the FFT which produces the spectrum. Figure 2 shows an example of multi-resolution sensing. A coarse resolution sensing is done by using a smaller $K_{1}$ size FFT with a resolution $f_{r 1}=\frac{B_{\text {tot }}}{K_{1}}$. The energy on each FFT bin $E_{i}$ for $i=0,1, \ldots K_{1}$ is compared with a threshold $t h_{1}$. We define $P e r$ as the percentage of the total number of bins where the energy are larger than $t h_{1}$. If this percentage is larger than a limit $p, P e r>p$, we assume the total band is too crowded to accommodate Cognitive Radio. If no bins have been found with significant energy (no $i$ where $E_{i}>t h_{1}$ ), namely $P e r=0$, we think the band is empty. In these two conditions, fine resolution sensing is not needed and Cognitive Radio will either start communication or wait for a licensed user to free the spectrum. Otherwise, Cognitive Radio will continue with fine resolution sensing with a resolution of $f_{r 2}$ to focus on those high energy bands (like the colored potion in figure 2) where licensed users are potentially active. However, the specific method to select the interested bands is not considered in our discussion. The interested portion of the spectrum is actually a part of the output bins of a larger $K_{2}$ size FFT, where $K_{2}=\frac{B_{t o t}}{f_{r 2}}$. Based on the result of fine resolution sensing, Cognitive Radio will determine the transmission scheme and wait for the next sensing cycle. A flowchart describing the multi-resolution sensing scheme is shown in figure 3 . The total cost of multi-resolution sensing can be expressed as:

$$
C_{\text {tot }}=\left\{\begin{array}{c}
C_{\text {coarse }} \text { others } \\
C_{\text {coarse }}+C_{\text {fine }} \text { if } 0<\text { Per }<p
\end{array}\right.
$$

, where $C$ denotes costs. Here, we make an important observation: only a portion of the larger FFT outputs is needed for fine resolution sensing. In this case, the naive implementation of a larger size FFT is inefficient. Therefore, an efficient algorithm which produces only a part of FFT outputs is desirable. Furthermore, there are several requirements for the efficient FFT implementation: 1) it can produce FFT outputs in any position depending on the need of Cognitive Radio; 2) it has reconfigurability from a normal FFT and vice versa; 3 ) it is easy to implement on hardware for real time processing.

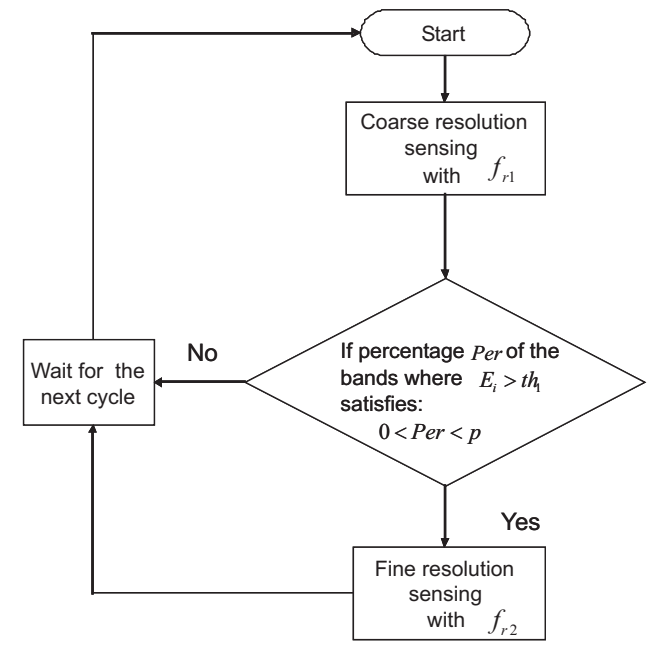

Fig. 3. Flowchart of multi-resolution sensing

\section{PROPOSED METHOD}

In [9], we proposed a sparse FFT for OFDM based Cognitive Radio where a large portion of subcarriers is switched off to avoid interference to licensed users by loading zeros. A sparse FFT is used in the OFDM receiver to demodulate the data in the nonzero part of the spectrum occupied by Cognitive Radio. We think the sparse FFT [9] can be also applied to the multi-resolution sensing system proposed in the previous section because it fulfills all aforementioned requirements.

Let us revisit the sparse FFT here. In mathematical terms, arrays or matrices where most of the elements have the same value (called the default value, usually 0 ) and only a few elements have a non-default value are sparse. It is beneficial and often necessary to take advantage of the sparse structure algorithmically to reduce the operations of the standard algorithms. The proposed sparse FFT is modified from transform decomposition in [10]. The basic idea is to divide a large FFT into two smaller size FFTs, which offers the opportunity to reduce computation. We explain the algorithm in details here. The DFT is defined as:

$$
X(k)=\sum_{n=0}^{N-1} x(n) W_{N}^{n k} k=0,1, \ldots, N-1
$$

, where $W_{N}^{n k}=e^{\frac{-j 2 \pi n k}{N}}$. We consider the case where $\mathrm{L}$ outputs are nonzero. Let $N$ be factorized as two integers $N_{1}$ and $N_{2}$, so $N=N_{1} N_{2}$. To facilitate the implementation, we make the size of FFT $N$ a power-of-two integer. We choose $N_{1}$ as the nearest power-of-two integer larger than $L$ and as a factor of $N$, denoted as $N_{1}=\lceil L\rceil_{\text {pow } 2}$. Therefore, $N, N_{1}$ and $N_{2}$ are all power-of-two integers. The index $n$ can be written as:

$$
\begin{gathered}
n=N_{2} n_{1}+n_{2} \\
n_{1}=0,1, \ldots, N_{1}-1 \quad n_{2}=0,1, \ldots, N_{2}-1
\end{gathered}
$$

Substitute $n$ in (2) with (3) and then the DFT can be rewritten 
as:

$$
\begin{aligned}
X(k) & =\sum_{n_{2}=0}^{N_{2}-1} \sum_{n_{1}=0}^{N_{1}-1} x\left(N_{2} n_{1}+n_{2}\right) W_{N}^{\left(N_{2} n_{1}+n_{2}\right) k} \\
& =\sum_{n_{2}=0}^{N_{2}-1}\left[\sum_{n_{1}=0}^{N_{1}-1} x\left(N_{2} n_{1}+n_{2}\right) W_{N}^{N_{2} n_{1} k}\right] W_{N}^{n_{2} k}
\end{aligned}
$$

We define:

$$
\begin{aligned}
X_{n_{2}}\left(\langle k\rangle_{N_{1}}\right) & =\sum_{n_{1}=0}^{N_{1}-1} x\left(N_{2} n_{1}+n_{2}\right) W_{N_{1}}^{n_{1} k} \\
& =\sum_{n_{1}=0}^{N_{1}-1} x_{n_{2}}\left(n_{1}\right) W_{N_{1}}^{n_{1} k}
\end{aligned}
$$

where \langle\rangle$_{N_{1}}$ denotes modulo $N_{1}$. So (4) can be written as:

$$
X(k)=\sum_{n_{2}=0}^{N_{2}-1} X_{n_{2}}\left(\langle k\rangle_{N_{1}}\right) W_{N}^{n_{2} k}
$$

The original $N$-point DFT with $L$ nonzero outputs is decomposed into two major parts: the $N_{2} N_{1}$-point DFTs in (5) which can be implemented as $N_{2} N_{1}$-point FFTs and the multiplications with twiddle factors and recombinations of the multiplications in (6). Because the index $k$ only consists of $L$ nonzero values, only $L$ twiddle factors are multiplied with each $X_{n_{2}}\left(\langle k\rangle_{N_{1}}\right)$ for $n_{2}=1,2, \ldots, N_{2}$. This multiplication part results in a computation reduction.

The number of complex multiplications in a $N$ point sparse FFT with $L$ nonzero outputs equals:

$$
M u l_{\text {sparse }}=\left(N_{2}-1\right) * L+\frac{N}{2} \log _{2} N_{1}
$$

From eq. 7, we can see the algorithm is more efficient for small $L$. The complexity increases with the number of non zeros $L$ and reaches the break even point when $L=\frac{N}{2}$. Since we set the constraints $N_{1}=\lceil L\rceil_{\text {pow } 2}$ and $N=N_{1} N_{2}$, the sparse FFT will be calculated as normal $N$ point FFT when $L>\frac{N}{2}$. These constraints are important modifications to the algorithm in [10] in the sense that they facilitate hardware implementations by exploiting power-of-two integers. In [10], the discussions are restricted to the case when only the first $L$ consecutive values are non-zeros. Our proposed algorithm can be applied to any non-zero distributions. Assuming the size of the FFT as $N=2048$ (a typical value in Cognitive Radio based IEEE 802.22), we compared the complexity of the sparse FFT and the radix-2 FFT in figure 4. The sparse FFT has less complexity than the radix-2 FFT when the nonzero ratio $\frac{L}{N}<0.5$ and it becomes even more efficient with the decrease of the nonzero ratio.

Let us consider a concrete multi-resolution sensing example. First, a coarse resolution sensing is done by FFT-128 with a resolution of $\frac{B_{t o t}}{128}$. If Cognitive Radio finds $5 \%$ of the total bandwidth needs fine resolution sensing, the system is reconfigured to the sparse FFT-2048 with the resolution of $\frac{B_{t o t}}{2048}$ (16 times finer) to only focus on those interested bands. In this case, the total cost of multi-resolution sensing is the

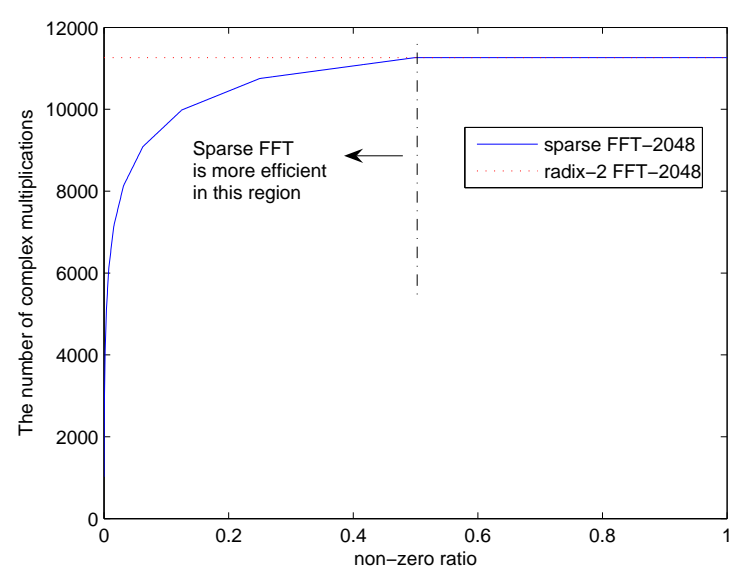

Fig. 4. Complexity comparison of the sparse FFT

complexity of the sparse FFT-2048 with 0.05 nonzero ratio plus the complexity of the radix-2 FFT-128, totally about 9000 complex multiplications. Comparing this with the fixed resolution sensing by the radix-2 FFT-2048, it gives about $20 \%$ saving. However, this saving will diminish with the increasing percentage of the bandwidth which requires fine resolution sensing. The break even point in our example is about $25 \%$ of the total bandwidth which requires fine resolution sensing. Beyond this point, the complexity of the radix-2 FFT-128 and sparse FFT-2048 will exceed the complexity of the radix-2 FFT-2048. Clearly, the condition to apply the proposed multiresolution sensing method is that only a small fraction of total bandwidth requires fine resolution sensing.

\section{IMPLEMENTATION}

The core of the proposed multi-resolution sensing architecture is a reconfigurable FFT which has to constantly switch from radix-2 FFT to the sparse FFT and vice versa. The reconfiguration has to be done in real-time with minimum overhead. The computational structure of the sparse FFT is presented in figure 5. The sparse FFT decomposes the DFT into two major parts: $N_{2}$ blocks of $N_{1}$-point radix-2 FFTs and the multiplications with twiddle factors together with the recombination of the multiplications. We can find the regularity in the memory addressing: a constant hop from the same position in one block to another (e.g. from $x_{0}(0)$ to $\left.x_{1}(0)\right)$. The normal radix-2 FFT is reusable in the first part of the sparse FFT. These interesting properties of the sparse FFT can be exploited to make efficient and reconfigurable hardware solutions.

As an experiment, we made an implementation of the dynamic reconfigurable FFT on coarse-grained reconfigurable hardware [11] developed in our group. The coarse-grained reconfigurable hardware named Montium [12] can implement concurrent processing with minimum control overhead. It has a low silicon cost, as the core is very small. For instance, the silicon area of a single Montium TP with $10 \mathrm{~KB}$ of embedded SRAM is $2.4 \mathrm{~mm}^{2}$ in $0.13 \mu \mathrm{m}$ CMOS technology. The 


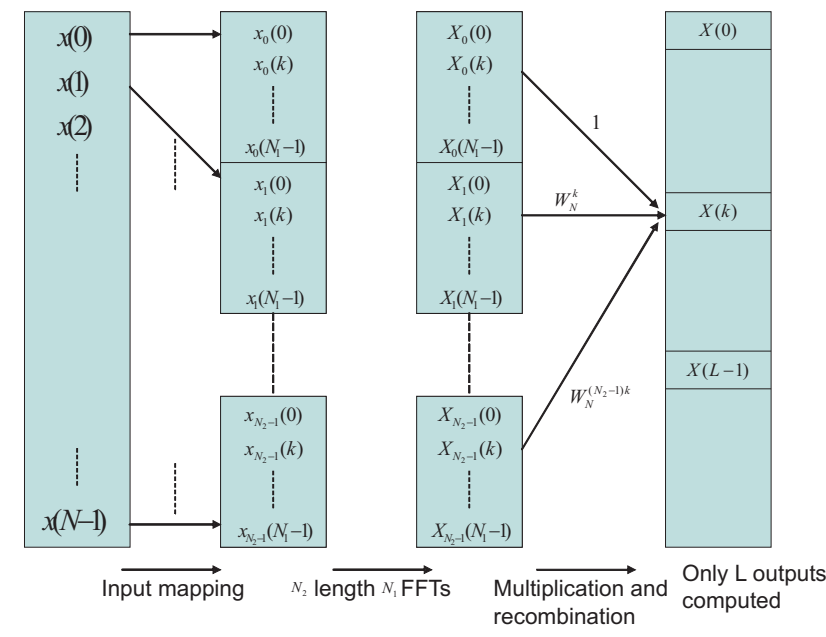

Fig. 5. Computational structure of the sparse FFT

power consumption in this technology is only $577 \mu \mathrm{W} / \mathrm{MHz}$ (including all memory accesses). The Montium configuration for the reconfigurable FFT is generated by using a Montium compiler using an FFT script. During the initialization stage we load the configuration of the largest radix-2 FFT (FFT512 in this experiment) needed into the Montium sequencer. The configuration in the sequencer defines instructions and contains a control part which is used to implement a sequencing state machine. Run-time reconfiguration is achieved by altering the configuration memory. Since smaller size FFTs can be reconstructed from a larger FFT by skipping FFT stages, switching to small FFTs only involves adapting a small part of the configuration. The radix-2 FFT configuration can be reused in the first part of sparse FFT, only the configuration code for multiplications with twiddle factors and recombination has to be added. Table I shows the costs of the reconfiguration for both radix-2 FFT and sparse FFT (less than 64 nonzeros) in terms of bytes. When using reconfigurable code, a large configuration is sent to the Montium at initialization and changing the FFT size involves small pieces of reconfiguration code. When using static code, reconfiguring FFT size requires a complete reconfiguration. Dynamic reconfiguration is about 10 times more efficient than reloading the complete static configuration.

\begin{tabular}{|c|c|c|}
\hline & reconfigurable code & static code \\
\hline Initialization & 1792 & 0 \\
\hline to 256-point & 82 & 1054 \\
\hline to 128-point & 78 & 970 \\
\hline to 64-point & 80 & 886 \\
\hline to 512-sparse (less than 64 point) & 78 & - \\
\hline
\end{tabular}

TABLE I

BYTES THAT NEED TO BE SENT FOR RECONFIGURATION

\section{CONCLUSION}

In this paper, we present an energy based multi-resolution sensing method. An efficient reconfigurable FFT module is the essence of this method. By reconfiguring from a small size radix-2 FFT to a large sparse FFT [9], only bands which are interested for Cognitive Radio are produced in a finer resolution. Our proposed method is beneficial in the following aspects: 1) it avoids sensing the spectrum with the fixed maximum frequency resolution which is not always necessary; 2) there is no need to tune the analog front-end to focus on the interesting bands for fine resolution sensing; 3) sparse FFT is used for a smart fine resolution sensing which only computes the interested bands in a finer resolution; 4) due to the regular computational structure of sparse FFT, it is easy to make a hardware implementation of the reconfigurable FFT module with very small reconfiguration overhead. 5) multi-resolution sensing based on sparse FFT can be easily integrated with the OFDM based Cognitive Radio in [9].

\section{ACKNOWLEDGMENT}

The work is sponsored by the Dutch Ministry of Economic affairs Freeband AAF project. We acknowledge the support for the Montium development tool by Recoresystems. The authors would like to thank their colleagues from the International Research Centre for Telecommunications-transmission and radar (IRCT) of the Technical University Delft (TUD) and the Signal and System (SAS) group at the University of Twente (UT) for the fruitful discussions in the AAF project.

\section{REFERENCES}

[1] J. Mitola III. Cognitive Radio: An Integrated Agent Architecture for Software Defined Radio, PhD Thesis, Royal Institute of Technology, Sweden, May. 2000.

[2] T.A. Weiss and F.K. Jondral, "Spectrum pooling: An innovative strategy for the enhancement of spectrum efficiency," IEEE Commun. Mag., Mar. 2004.

[3] H. Urkowitz, "Energy detection of unkown deterministic signals," Proc. of IEEE, April 1967.

[4] W.A.Gardner,"Signal interception: A unifying theoretical framework for feature detection," IEEE Trans. on Communications, August 1988.

[5] D. Cabric, S. M. Mishra, R. W. Brodersen, "Implementation issues in spectrum sensing for Cognitive Radios," Asilomar Conference on Signals, Systems, and Computers, 2004.

[6] S.M. Mishra, A. Sahai, R. W. Brodersen, "Cooperative sensing among Cognitive Radios," International Conference on Communications (ICC), Istanbul, June, 2006.

[7] Y. Hur, J. Park, W. Woo, K. Lim, C.-H. Lee, H.S. Kim, and J. Laskar, "A wideband analog multi-resolution spectrum sensing (MRSS) technique for cognitive radio (CR) systems," International Symposium on Circuits and Systems (ISCAS), May, 2006.

[8] Nathan M. Neihart, Sumit Roy, and David J. Allstot, "A Parallel, MultiResolution Sensing Technique for Multiple Antenna Cognitive Radios," International Symposium on Circuits and Systems (ISCAS), May, 2007.

[9] Qiwei Zhang, Andre B.J. Kokkeler and Gerard J.M. Smit "An efficient FFT for OFDM based Cognitive Radio on a reconfigurable architecture," IEEE International Conference on Communications (ICC2007), 2007, UK

[10] Henrik V. Sorensen and Sidney Burrus, "Efficient computation of the DFT with only a subset of input or output points," IEEE Trans. on Signal Processing, Mar. 1993

[11] Gerard K. Rauwerda, Paul M. Heysters and Gerard J.M. Smit "Towards software defined radios using coarse-grained reconfigurable hardware," IEEE Transcation on Very Large Scale Integration Systems, Jan. 2008

[12] Paul Heysters Coarse-Grained Reconfigurable Processors; Flexibility meets Efficiency, PhD Thesis, University of Twente, Sep. 2004 